\title{
On the Size-Dependent Elasticity of Penta-Twinned Silver Nanowires
}

\author{
Tzu-Hsuan Chang", Guangming Cheng", Chengjun Li and Yong Zhu*
}

\begin{abstract}
Penta-twinned metallic NWs have recently received much attention due to their excellent mechanical properties. However, their elasticity size effect remains not well understood due to the large discrepancy in the reported experimental and simulation results. This paper reports an experimental effort to address the discrepancy about the size-dependent Young's modulus of penta-twinned Ag NWs. Two independent experiments on the same NW, in-situ resonance test and tensile test in a scanning electron microscope, were used to measure the Young's moduli. The cross-sectional shape of the Ag NWs was found to transit from pentagon to circle with decreasing NW diameter, which can modify the Young's modulus as much as $8 \%$ (for resonance test) and $19 \%$ (for tensile test) for the tested diameter range. This work confirmed that the Young's modulus of penta-twinned Ag NWs increases with decreasing NW diameter.

\# These authors contributed equally to this work.

* To whom correspondence should be addressed. E-mail: yong_zhu@ncsu.edu
\end{abstract}




\section{Introduction}

Recent advance in nanotechnology has brought forth a host of nanomaterials, such as nanoparticles, nanowires (NWs), nanotubes and 2D nanomaterials that exhibit ultrahigh mechanical strength [1], [2]. Such nanomaterials not only are building blocks for a broad range of nanomaterial-enabled applications, but also provide ideal platforms for studying fundamental mechanical behaviors at the nanoscale. As an example, metallic NWs have shown promising potential for flexible/transparent electronics and stretchable electronics [3], [4]; their deformation mechanism is now known to transit from forest dislocation dynamics to dislocation nucleation from free surfaces [5]-[7].

A variety of metallic NWs can be synthesized now using several methods including electrochemical deposition [8] and physical vapor deposition [9], [10]. Among all the metallic NWs, penta-twinned NWs are unique in microstructure - each NW has five twin segments joined along a common quintuple line in the axial direction. In addition, synthesis of pentatwinned metallic NWs based on solution phase is relatively easy and scalable [11]. Pentatwinned metallic NWs have recently received much attention due to their excellent mechanical properties. For instance, increased Young's modulus and yield strength with decreasing NW diameter have been recently reported [6], [12]. More recently, recoverable plasticity [13], [14] and strain hardening [15] have been investigated.

However, the elasticity size effect of penta-twinned metallic NWs is still under debate. Both experimental and modeling results on the Young's modulus of penta-twinned Ag NWs, as an example, exhibited large discrepancies. Using in-situ tensile tests in scanning or transmission electron microscopy (SEM/TEM), Zhu et al. [6] and Filleter et al. [12] reported pronounced stiffening effect, i.e., increased Young's modulus with decreasing NW diameter. Using bending 
tests under atomic force microscopy (AFM), Jing et al. [16] found a similar stiffening effect to the above tensile tests. In contrast, Wu et al. [17] obtained an average Young's modulus that is higher than the bulk value but independent of the NW diameter; Chen et al. [18] reported Young's moduli that are higher than the bulk value but without obvious size effect, similar to the result by Alducin et al. [19] using in-situ TEM bending. In all the experiments above, the NWs were $<110>$ oriented with diameters typically between 20 and $140 \mathrm{~nm}$. However, no experiments compared the Young's moduli of the penta-twinned Ag NW and the single-crystalline counterpart.

Atomistic simulations showed pronounced stiffening size effect but no significant effect of the penta-twinned microstructure compared to the single-crystalline counterpart [20], [21]. Other atomistic simulations revealed the similar size effect, but also showed strong effect of the pentatwinned microstructure, leading to higher Young's modulus than that of the single-crystalline NW [22], [23]. The microstructure effect was attributed to the high compressive at the core of the penta-twinned NW. Similar conclusions on the size and microstructure effects were observed in other penta-twinned FCC NWs, such as $\mathrm{Cu}, \mathrm{Au}, \mathrm{Ni}, \mathrm{Pd}$ and etc [24], [25]. More recently, Bizek and co-workers carried out a systematic study on the Young's modulus of several types of FCC penta-twinned NWs using atomistic simulations and analytical modeling [26]. They found that while the size effect is atomic origin (e.g., surface stress and surface elasticity), the effect of the penta-twinned microstructure is due to compatibility constraint imposed by the microstructure and elastic anisotropy of the FCC metal.

The elasticity size effect can be generally attributed to two mechanisms [27]: surface elasticity [28]-[32] and bulk nonlinear elasticity (as a result of the surface stress) [33]. Under different loading modes, the elasticity size effect manifests differently for different mechanisms 
[21], [28], [34]. For instance, in the case of surface elasticity, the elasticity size effect would be stronger under bending than under tension as the surface plays a more important role under bending. More specifically, the NW Young's modulus $E=E_{c}+8 \frac{S}{D}$ and $E=E_{c}+4 \frac{S}{D}$, respectively, under bending and tension, where $E_{c}$ is the Young's modulus of the core and $D$ is the NW diameter [34]. Therefore, for probing the underlying mechanism of the elasticity size effect, it is valuable to obtain the Young's modulus under different loading modes. Zhu and coworkers measured the elasticity size effect of $\mathrm{ZnO}$ NWs under both tension and buckling [34]. However, since the buckling force and hence the measured Young's modulus sensitively depend on the NW diameter $\left(4^{\text {th }}\right.$ power in contrast to square in the case of tension), the buckling method could lead to larger error in measuring the Young's modulus compared to other methods such as tension and resonance [35].

This paper reports an experimental effort to address the discrepancy about the size-dependent Young's modulus of penta-twinned Ag NWs. Two independent experiments on the same NW, in-situ SEM resonance test and tensile test, were used to measure the Young's moduli. In addition, the cross-sectional shape of the Ag NWs was measured as a function of the NW diameter, which was found to transit from pentagon to circle with decreasing NW diameter. The effect of the cross-sectional shape on the measured Young's modulus was evaluated. This work confirmed that the Young's modulus of penta-twinned Ag NWs increases with decreasing NW diameter, though the size effect is less pronounced compared to our previous result [6].

\section{Materials and Methods}

Penta-twinned Ag NWs were synthesized by reducing $\mathrm{AgNO}_{3}$ with ethylene glycol in the presence of polyvinyl pyrrolidone. More details of the NW synthesis process are provided 
elsewhere [6], [11]. The solution of Ag NWs was diluted with deionized water and then purified by centrifugation.

In-situ resonance and tension tests of the same NWs were carried out inside a dual beam SEM/FIB system (FEI Quanta 3D FEG) using a two-probe setup. The first probe was glued on a piezoelectric sheet that was used to provide mechanical vibration. The second probe was installed on a nanomanipulator (Klocke Nanotechnik, Germany) for manipulating an individual NW including picking up from substrate and mounting onto a MEMS stage. After the NW was transferred from the second to the first probe and clamped using electron beam induced deposition of platinum (EBID-Pt), the piezoelectric sheet was excited into mechanical vibration (Figure 1a). Note that the clamping condition was inspected carefully following [36] in order to obtain the Young's modulus accurately. As soon as the frequency of AC signal was close to the resonance of NW, the vibration amplitude of NW increased sharply, as shown in Figure $1 \mathrm{~b}$. Around the resonance frequency of the NW, SEM images of the vibrating NW were taken at a number of frequencies, from which the vibration amplitude was measured as a function of the frequency (Figure 2a). Then the resonance frequency can be determined from the amplitudefrequency plot. After the resonance test, the NW was transferred from the first probe back to the second probe (attached to the nanomanipulator) for the in-situ tensile testing. For more details of transferring a NW, please see Supporting Information.

Prior to the tensile testing, the NW on the second probe will be further transferred to a microelectromechanical system (MEMS) stage following [37]. The MEMS stage consists of a thermal actuator, a capacitive load sensor and a gap in between where the NW will be mounted. The MEMS stage was fabricated at MEMSCAP (Durham, NC) using the Silicon-on-Insulator 
Multi-User MEMS Processes (SOI-MUMPs). The strain rate was nominally $10^{-4} \mathrm{~s}^{-1}$. Details of performing in-situ tensile testing using the MEMS stage can be found elsewhere [13], [38].

Cross-section TEM samples were prepared by embedding Ag NWs into Gatan G1 epoxy with a $\varphi 3 \mathrm{Cu}$ tube, cutting the specimen discs with a thickness of $\sim 0.5 \mathrm{~mm}$, mechanically polishing with Allied Multiprep System and finally ion milling the sample via Gatan 791 PIPS while cooling with liquid nitrogen.

\section{Results and Discussion}

From Figure 2a, the first resonance frequency of the penta-twinned $\mathrm{Ag} \mathrm{NW}$ was identified as $799 \mathrm{kHz}$, which was then converted to the Young's modulus assuming a pentagonal cross section of the Ag NW. According to a simple beam theory, the $n$th mode resonance frequency of a cantilevered beam is

$$
{ }_{n}=\frac{n^{2}}{L^{2}} \sqrt{\frac{E I}{A}}
$$

where $E$ is the Young's modulus, $I$ is the moment of inertia, $L$ is the beam length, $A$ is the crosssectional area and $\rho$ is the beam density. $\beta_{0}=1.875, \beta_{1}=4.694$ and $\beta_{2}=7.855$ correspond to the first three resonance modes for a cantilevered beam [36]. The Young's modulus was calculated to be $101.8 \mathrm{GPa}$ according to Eq. (1).

Figure $2 \mathrm{~b}$ shows the stress-strain curve from the tensile test of the same penta-twinned Ag NW that was characterized in the resonance test. Here again a pentagonal cross section was assumed in calculating the stress. In all our tensile tests, penta-twinned Ag NWs showed clearly linear elastic behavior up to $\sim 1.5 \%$ strain, followed with nonlinear elastic behavior and strain hardening. The Young's modulus was extracted from the stress-strain curve as the slope of the linear elastic regime. 
Figure 3a plots the measured Young's moduli of the penta-twinned Ag NWs using both the resonance and tensile tests. Note that in all cases, pentagonal cross sections were assumed. 13 tensile data points that were obtained previously using an AFM cantilever as the load sensor were included [6]. When the NW diameter is larger than $\sim 70 \mathrm{~nm}$, the Young's modulus is approximately constant close to the bulk value ( $84 \mathrm{GPa})$. But when the $\mathrm{NW}$ diameter is smaller than $\sim 70 \mathrm{~nm}$, a strong stiffening size effect can be seen (i.e., the Young's modulus increases with decreasing NW diameter). The size effect as shown in Figure 3a is similar to that reported by Jing et al. [16]. The stiffening trend is also in agreement with that of Filleter et al. [12] but our size effect is stronger than theirs in magnitude. A notable difference is that we used pentagonal cross section in Figure 3a, but Filleter et al. used circular cross section in their work. Apparently the cross-sectional shape affects how the cross-sectional area and moment of inertia are calculated, and thus affects the Young's modulus values obtained from the tensile and resonance tests.

Cross-sectional samples of penta-twinned Ag NWs of different diameters were prepared and observed in TEM to determine their cross-sectional shapes. Figure 4a shows the cross-sectional TEM images of several penta-twinned $\mathrm{Ag} \mathrm{NWs}$ of different diameters. For relatively large diameters (larger than $100 \mathrm{~nm}$ or so), the cross sections generally appear to be pentagonal in shape with blunted vertices. With decreasing diameter, the blunt vertices take more percentage along the perimeter and as a result the cross section appears more like a circle. NWs typically possess well-developed surface facets with low surface energies. For FCC crystal, the surface energy increases from $\{111\}$ to $\{100\}$ to $\{110\}$ planes [39]. In the case of penta-twinned NWs, the five facets correspond to $\{100\}$ planes as a result of the unique penta-twin microstructure. On the other hand, circular surface is effective in minimizing the surface area and thus the surface 
energy. Therefore, the blunted vertices arise as a result of the competition of the surface faceting and surface area minimization.

However, in our calculations so far all the cross sections were assumed to be pentagonal regardless of the NW diameter. Hence it is necessary to re-evaluate the cross-sectional area as a function of the NW diameter. Figure $4 \mathrm{~b}$ shows the cross-sectional areas measured directly from the TEM images (square dots) versus the NW diameters; the NW diameter (d) was defined as the largest distance between any two points on the cross section in the horizontal direction of the cross-sectional image (also the largest distance as measured in the SEM image during the mechanical testing). For the purpose of comparison, the areas of ideal pentagon and circle were included in Figure 4b too (dashed lines). As expected, the measured cross-sectional areas agree quite well with the circular area and pentagonal area, respectively, for relatively small and large NW diameters. The measured cross-sectional area can be fitted with the following function

$$
A=\frac{d d_{1}}{d_{2} d_{1}} A_{\text {circle }}+\frac{d_{2} d}{d_{2} d_{1}} A_{\text {pentagon }}
$$

where $d_{1}=30 \mathrm{~nm}$ and $d_{2}=170 \mathrm{~nm}$ define the range of the diameters where the fitting was applied, $A_{\text {circle }}=\mathrm{d}^{2} / 4$ and $A_{\text {pentagon }}=5 \mathrm{~d}^{2} / 8 \sin 72^{\circ}$ are the areas of an ideal circle and pentagon, respectively. Eq. (2) fitted the measured cross-sectional areas very well.

A similar form was assumed for

$$
\frac{I}{A}=\frac{d}{d_{2}} d_{1} d_{1} \frac{I_{\text {circle }}}{A_{\text {circle }}}+\frac{d_{2} d}{d_{2} d_{1}} \frac{I_{\text {pentagon }}}{A_{\text {pentagon }}}
$$

where $I_{\text {circle }}=\pi d^{4} / 64$ and $I_{\text {pentagon }}=\frac{A d^{2}}{192 \sin ^{2}\left(54^{\circ}\right)}\left[1+3 \cot ^{2}\left(\frac{180^{\circ}}{5}\right)\right][40]$ are the moments of inertia of an ideal circle and pentagon, respectively. 
With the fitting functions, Eqs (2) and (3), the obtained Young's modulus can be corrected. The correction is more pronounced for the tensile test. When the cross section is changed from pentagon to circle, the Young's moduli obtained from the tensile test and resonance test reduce as much as $19 \%$ and $8 \%$, respectively. Figure $3 \mathrm{~b}$ plots the corrected Young's moduli of the penta-twinned Ag NWs using both the resonance and tensile tests. After the correction, a less pronounced stiffening size effect was obtained. When the diameter is larger than $\sim 70 \mathrm{~nm}$, a nearly constant Young's modulus can be seen, slightly smaller than the bulk value (84 GPa); when the diameter is less than $\sim 70 \mathrm{~nm}$, the Young's modulus increases with decreasing diameter. The size effect is in good agreement with that reported by Filleter et al. [12]. This agreement is not surprising because the circular cross section was assumed for the NWs in their work. However, our data are much more systematic compared to the only three data points in their work showing the size effect. A plot of summarizing our data and those reported in the literature is shown in Figure S3.

Very similar size effects were observed in both resonance and tension tests. It appears that under resonance the size effect is slightly stronger. For example, for those NWs where both resonance and tension were performed on the same NWs, the differences between the modulus values in both resonance and tension can be seen in Figure S4. It is known that the loadingmode-dependent size effect is postulated to be a result of differences in NW surface and core Young's moduli. In general, for metal NWs, the surface stress is tensile and as a result the NW core is in compression. In the case of a penta-twinned NW, five twin segments are joined along a common quintuple line with a disclination $\left(7.35^{\circ}\right)$. Such a disclination results in substantial compressive stress in the NW core and tensile stress close to the surface in the NW axial 
direction [13], [26]. While this additional compressive stress leads to higher Young's modulus in the core, the distribution of the Young's modulus in the radial direction is rather complicated.

Apparently some combination of core nonlinear elasticity and surface elasticity is responsible for the observed size effect in Young's modulus of the penta-twinned Ag NWs. In view that under resonance the size effect is slightly stronger, surface elasticity plays a slightly more important role. But it is difficult to separate the two effects. Our results agree well with the atomic simulations of $\langle 110\rangle$-oriented Ag NWs under both tension and bending, where the stiffening size effect was attributed to both core nonlinear elasticity and surface elasticity [21].

For the large NW diameters, the measured Young's moduli are close to the bulk value for both resonance and tension. Bitzek and co-workers predicted that the Young's modulus at relatively large diameter should be higher than the value for single-crystalline $\mathrm{Ag}$ in the $<110\rangle$ direction (84 GPa) as a result of the anisotropic elasticity and compatibility constraint imposed by the penta-twinned structure [26]. More specifically, elastic anisotropy leads to different Poisson's ratios in the directions orthogonal to the tensile axis. The constraint of constant wedge angle imposed by the penta-twinned structure effectively increases the Young's modulus. However, in their work every twin segment was assumed to possess the same wedge angle and this angle remain constant during the tension of the NW. In reality, defects such as interfacial dislocations and stacking faults might exist near some of the twin boundaries (Figure 5). Such defects could relax the compatibility constraint, which might account for the fact that the measured Young's moduli at large NW diameter are close to the bulk value, smaller than the value predicted by Bitzek and co-workers. However, the exact effect of the interfacial dislocations and stacking faults on the elasticity of penta-twinned NWs is not known yet and warrants further investigation. 


\section{Conclusions}

Penta-twinned metallic NWs have recently received much attention due to their excellent mechanical properties. However, their elasticity size effect remains not well understood due to the large discrepancy in the reported experimental and simulation results. This paper reports an experimental effort to address the discrepancy about the size-dependent Young's modulus of penta-twinned Ag NWs. Two independent experiments on the same NW, in-situ SEM resonance test and tensile test, were used to measure the Young's moduli. The cross-sectional shape of the Ag NWs was found to transit from pentagon to circle with decreasing NW diameter, which can modify the Young's modulus as much as $8 \%$ (for resonance test) and 19\% (for tensile test) for the NW diameter range used in this work. This work confirmed that the Young's modulus of penta-twinned Ag NWs increases with decreasing NW diameter.

The penta-twinned microstructure leads to not only some very interesting and highly desirable mechanical properties such as recoverable plasticity and strain hardening, but also complicated stress state and defect structures, which make the analysis and interpretation of their mechanical behaviors including elasticity and plasticity quite a challenge. For future work, detailed microstructure characterization, e.g., via cross-sectional TEM, should be taken into account.

\section{References:}

[1] T. Zhu and J. Li, “Ultra-strength materials," Prog. Mater. Sci., vol. 55, no. 7, pp. 710-757, Sep. 2010.

[2] J. R. Greer and J. T. M. De Hosson, "Plasticity in small-sized metallic systems: Intrinsic versus extrinsic size effect,” Prog. Mater. Sci., vol. 56, no. 6, pp. 654-724, Aug. 2011. 
[3] F. Xu and Y. Zhu, "Highly Conductive and Stretchable Silver Nanowire Conductors," Adv. Mater., vol. 24, no. 37, pp. 5117-5122, 2012.

[4] S. Yao and Y. Zhu, "Nanomaterial-Enabled Stretchable Conductors: Strategies, Materials and Devices," Adv. Mater., vol. 27, no. 9, pp. 1480-1511, 2015.

[5] T. Zhu, J. Li, A. Samanta, A. M. Leach, and K. Gall, "Temperature and Strain-Rate Dependence of Surface Dislocation Nucleation," Phys. Rev. Lett., vol. 100, no. 2, p. 025502, Jan. 2008.

[6] Y. Zhu, Q. Qin, F. Xu, F. Fan, Y. Ding, T. Zhang, B. J. Wiley, and Z. L. Wang, "Size effects on elasticity, yielding, and fracture of silver nanowires: In situ experiments," Phys. Rev. B, vol. 85, no. 4, p. 045443, Jan. 2012.

[7] L. Y. Chen, M. He, J. Shin, G. Richter, and D. S. Gianola, "Measuring surface dislocation nucleation in defect-scarce nanostructures," Nat. Mater., no. May, pp. 1-8, 2015.

[8] D. Jang, X. Li, H. Gao, and J. R. Greer, "Deformation mechanisms in nanotwinned metal nanopillars.," Nat. Nanotechnol., vol. 7, no. 9, pp. 594-601, Sep. 2012.

[9] G. Richter, K. Hillerich, D. S. Gianola, R. Mönig, O. Kraft, and C. A. Volkert, "Ultrahigh strength single crystalline nanowhiskers grown by physical vapor deposition.," Nano Lett., vol. 9, no. 8, pp. 3048-52, Aug. 2009.

[10] J.-H. Seo, Y. Yoo, N.-Y. Park, S.-W. Yoon, H. Lee, S. Han, S.-W. Lee, T.-Y. Seong, S.-C. Lee, K.-B. Lee, P.-R. Cha, H. S. Park, B. Kim, and J.-P. Ahn, "Superplastic deformation of defect-free Au nanowires via coherent twin propagation.," Nano Lett., vol. 11, no. 8, pp. 3499-502, Aug. 2011.

[11] B. Wiley, Y. Sun, B. Mayers, and Y. Xia, "Shape-controlled synthesis of metal nanostructures: the case of silver," Chem. Eur. J., vol. 11, no. 2, pp. 454-463, 2005.

[12] T. Filleter, S. Ryu, K. Kang, J. Yin, R. A. Bernal, K. Sohn, S. Li, J. Huang, W. Cai, and H. D. Espinosa, "Nucleation-controlled distributed plasticity in penta-twinned silver nanowires.," Small, vol. 8, no. 19, pp. 2986-93, Oct. 2012.

[13] Q. Qin, Y. Sheng, G. Cheng, X. Li, T.-H. Chang, G. Richter, Y. Zhu, and H. Gao, "Recoverable Plasticity in Penta-twinned Metallic Nanowires Governed by Dislocation Nucleation and Retraction," Nat. Commun., vol. 6, p. 5983, 2015.

[14] R. A. Bernal, A. Aghaei, S. Lee, S. Ryu, K. Sohn, J. Huang, W. Cai, and H. D. Espinosa, "Intrinsic Bauschinger Effect and Recoverable Plasticity in Penta-twinned Silver Nanowires Tested in Tension," Nano Lett., vol. 15, no. 1, pp. 139-146, 2015.

[15] S. Narayanan, G. Cheng, Z. Zeng, Y. Zhu, and T. Zhu, "Strain Hardening and Size Effect in Five-fold Twinned Ag Nanowires," Nano Lett., vol. 15, no. 6, pp. 4037-4044, 2015.

[16] G. Jing, H. Duan, X. Sun, Z. Zhang, J. Xu, Y. Li, J. Wang, and D. Yu, "Surface effects on elastic properties of silver nanowires: Contact atomic-force microscopy," Phys. Rev. B, vol. 73, no. 23, p. 235409, Jun. 2006.

[17] B. Wu, A. Heidelberg, J. J. Boland, J. E. Sader, Sun, and Li, "Microstructure-Hardened Silver Nanowires," Nano Lett., vol. 6, no. 3, pp. 468-472, Mar. 2006. 
[18] Y. Chen, B. L. Dorgan, D. N. McIlroy, and D. E. Aston, "On the importance of boundary conditions on nanomechanical bending behavior and elastic modulus determination of silver nanowires," J. Appl. Phys., vol. 100, no. 10, 2006.

[19] D. Alducin, R. Borja, E. Ortega, J. J. Velazquez-Salazar, M. Covarrubias, F. M. Santoyo, L. Baz??n-D??az, J. E. Sanchez, N. Torres, A. Ponce, and M. José-Yacamán, "In situ transmission electron microscopy mechanical deformation and fracture of a silver nanowire," Scr. Mater., vol. 113, pp. 63-67, 2016.

[20] M. T. McDowell, A. M. Leach, and K. Gall, "On the elastic modulus of metallic nanowires," Nano Lett., vol. 8, no. 11, pp. 3613-3618, 2008.

[21] M. T. McDowell, A. M. Leach, and K. Gall, "Bending and tensile deformation of metallic nanowires," Model. Simul. Mater. Sci. Eng., vol. 16, no. 4, p. 045003, 2008.

[22] J. H. Yoo, S. I. Oh, and M. S. Jeong, "The enhanced elastic modulus of nanowires associated with multitwins," J. Appl. Phys., vol. 107, no. 9, 2010.

[23] J. Y. Wu, S. Nagao, J. Y. He, and Z. L. Zhang, "Role of five-fold twin boundary on the enhanced mechanical properties of fcc Fe nanowires," Nano Lett., vol. 11, no. 12, pp. 5264-5273, 2011.

[24] Y. G. Zheng, Y. T. Zhao, H. F. Ye, and H. W. Zhang, "Size-dependent elastic moduli and vibrational properties of fivefold twinned copper nanowires.," Nanotechnology, vol. 25, no. 31, p. 315701, Aug. 2014.

[25] Y.-H. Wen, R. Huang, Z.-Z. Zhu, and Q. Wang, "Mechanical properties of platinum nanowires: An atomistic investigation on single-crystalline and twinned structures," Comput. Mater. Sci., vol. 55, pp. 205-210, Apr. 2012.

[26] F. Niekiel, E. Spiecker, and E. Bitzek, "Influence of anisotropic elasticity on the mechanical properties of fivefold twinned nanowires," J. Mech. Phys. Solids, vol. 84, pp. 358-379, Nov. 2015.

[27] H. S. Park, W. Cai, H. D. Espinosa, and H. Huang, "Mechanics of crystalline nanowires," MRS Bull., vol. 34, no. 03, pp. 178-183, 2009.

[28] R. E. Miller and V. B. Shenoy, "Size-dependent elastic properties of nanosized structural elements," Nanotechnology, vol. 11, no. 3, pp. 139-147, 2000.

[29] R. Dingreville, J. Qu, and M. Cherkaoui, "Surface free energy and its effect on the elastic behavior of nano-sized particles, wires and films," J. Mech. Phys. Solids, vol. 53, pp. 1827-1854, 2005.

[30] J. K. Diao, K. Gall, and M. L. Dunn, "Atomistic simulation of the structure and elastic properties of gold nanowires," J. Mech. Phys. Solids, vol. 52, no. 9, pp. 1935-1962, 2004.

[31] L. G. Zhou and H. C. Huang, "Are surfaces elastically softer or stiffer?," Appl. Phys. Lett., vol. 84, no. 11, pp. 1940-1942, 2004.

[32] P. Sharma, S. Ganti, and N. Bhate, "Effect of surfaces on the size-dependent elastic state of nano-inhomogeneities,” Appl. Phys. Lett., vol. 82, no. 4, pp. 535-537, 2003.

[33] H. Y. Liang, M. Upmanyu, and H. C. Huang, "Size-dependent elasticity of nanowires: Nonlinear effects," Phys. Rev. B, vol. 71, no. 24, p. 241403, 2005. 
[34] F. Xu, Q. Qin, A. Mishra, Y. Gu, and Y. Zhu, "Mechanical properties of ZnO nanowires under different loading modes," Nano Res., vol. 3, no. 4, pp. 271-280, Mar. 2010.

[35] Y. Zhu, "In-situ Nanomechanical Testing of Crystalline Nanowires in Electron Microscopes," JOM, vol. 68, no. 1, pp. 84-93, 2016.

[36] Q. Qin, F. Xu, Y. Cao, P. I. Ro, and Y. Zhu, "Measuring true Young's modulus of a cantilevered nanowire: effect of clamping on resonance frequency.," Small, vol. 8, no. 16, pp. 2571-6, Aug. 2012.

[37] Y. Zhu and H. D. Espinosa, "An electromechanical material testing system for in situ electron microscopy and applications.," Proc. Natl. Acad. Sci. U. S. A., vol. 102, no. 41, pp. 14503-8, Oct. 2005.

[38] G. Cheng, T.-H. Chang, Q. Qin, H. Huang, and Y. Zhu, "Mechanical properties of silicon carbide nanowires: effect of size-dependent defect density.," Nano Lett., vol. 14, no. 2, pp. 754-8, Feb. 2014.

[39] L. Vitos, A. V. Ruban, H. L. Skriver, and J. Kollár, "The surface energy of metals," Surf. Sci., vol. 411, no. 1-2, pp. 186-202, Aug. 1998.

[40] K. Rektorys, Survey of Applicable Mathematics. Cambridge, MA: The MIT Press, 1969. 


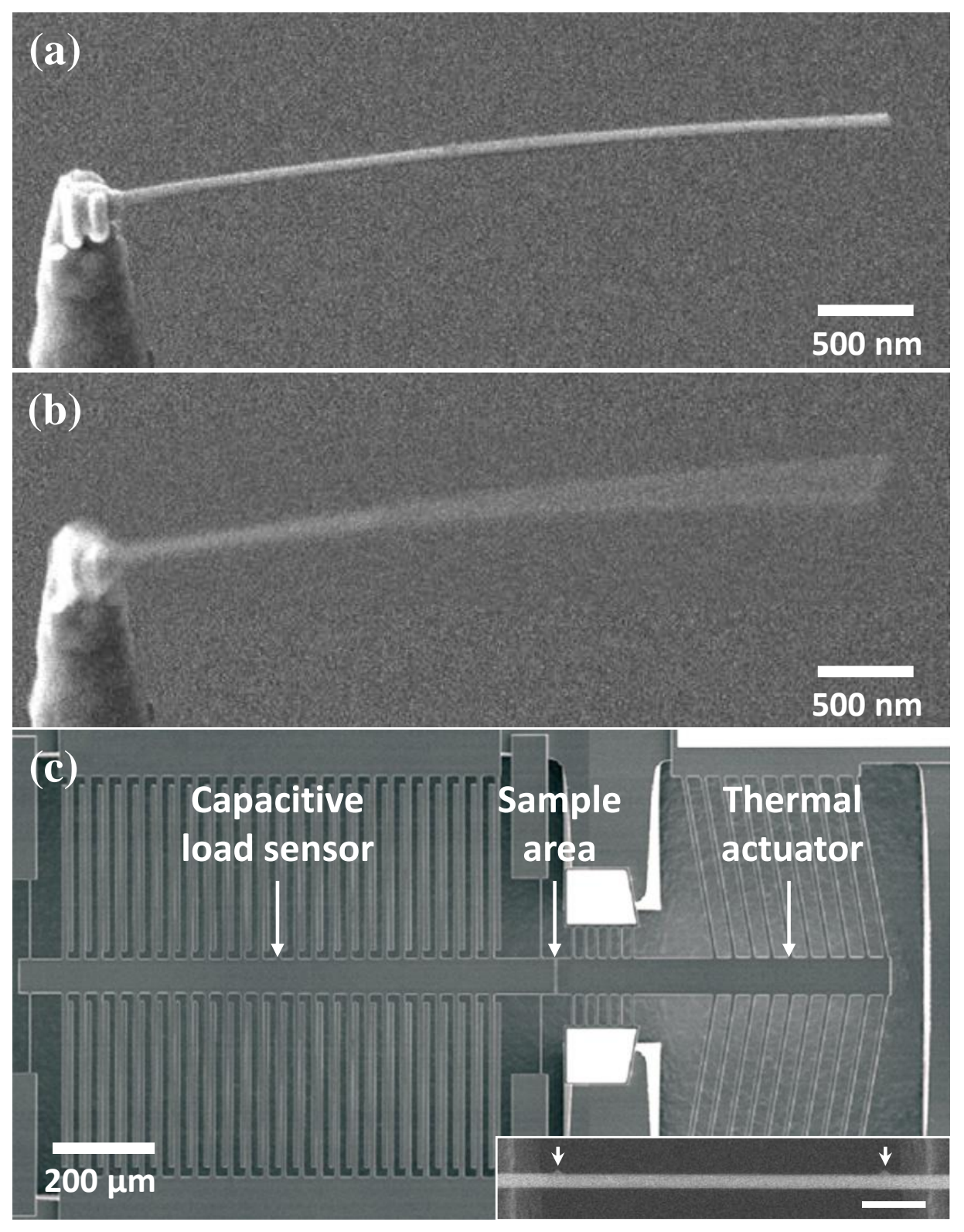

Figure 1. (a) SEM image of a penta-twinned Ag NW with one end welded on the tip of the tungsten probe. (b) SEM image of the same penta-twinned Ag NW under resonance. (c) SEM image of the MEMS testing stage with the same penta-twinned Ag NW. Inset shows the NW across the actuator and load sensor. The two small arrows indicate the deposited strain markers. Scale bar, $500 \mathrm{~nm}$. 

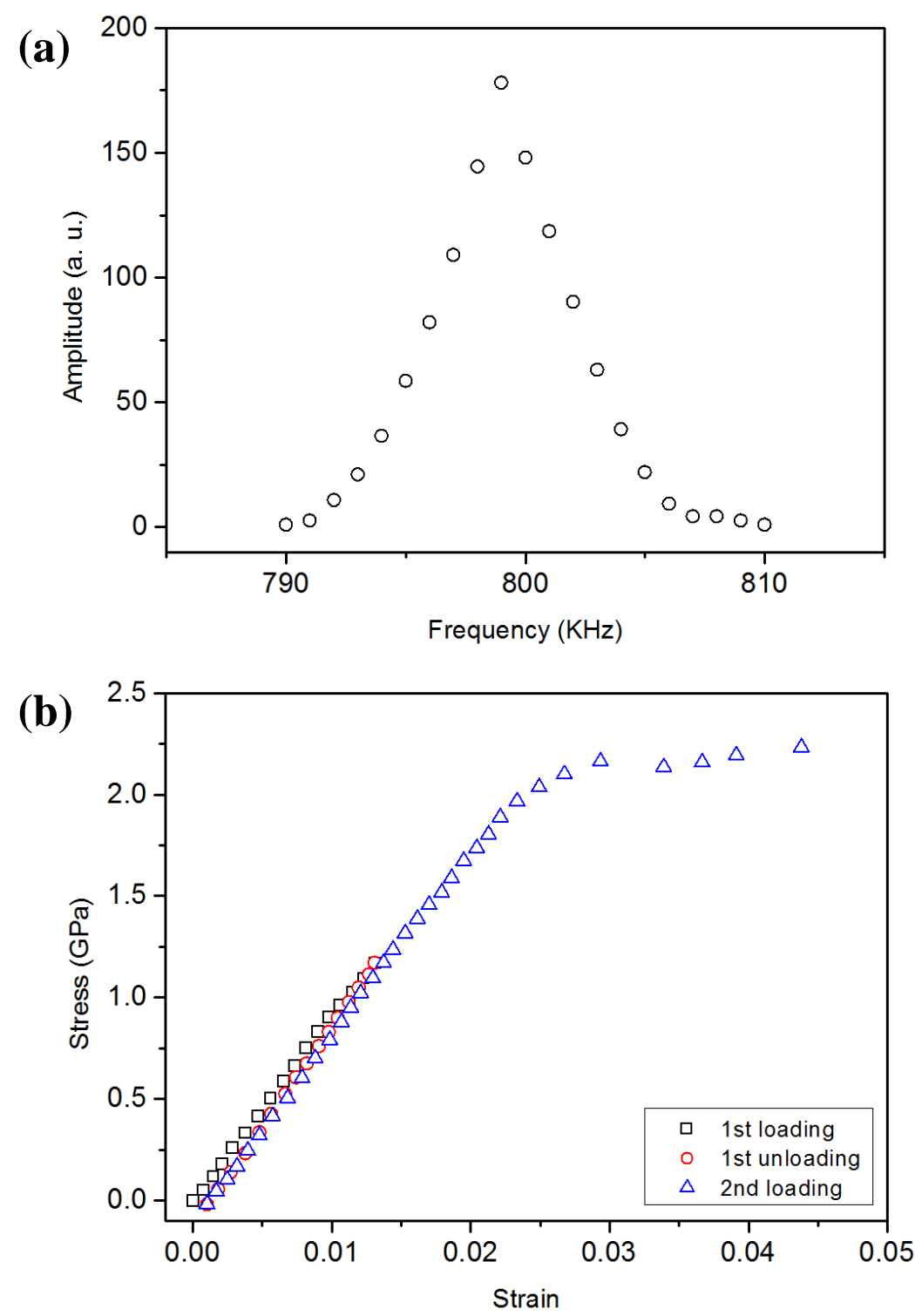

Figure 2. (a) The amplitude versus frequency plot of a penta-twinned Ag NW with diameter of $62 \mathrm{~nm}$. (b) Stress-strain of the same penta-twinned Ag NW under tensile loading. 

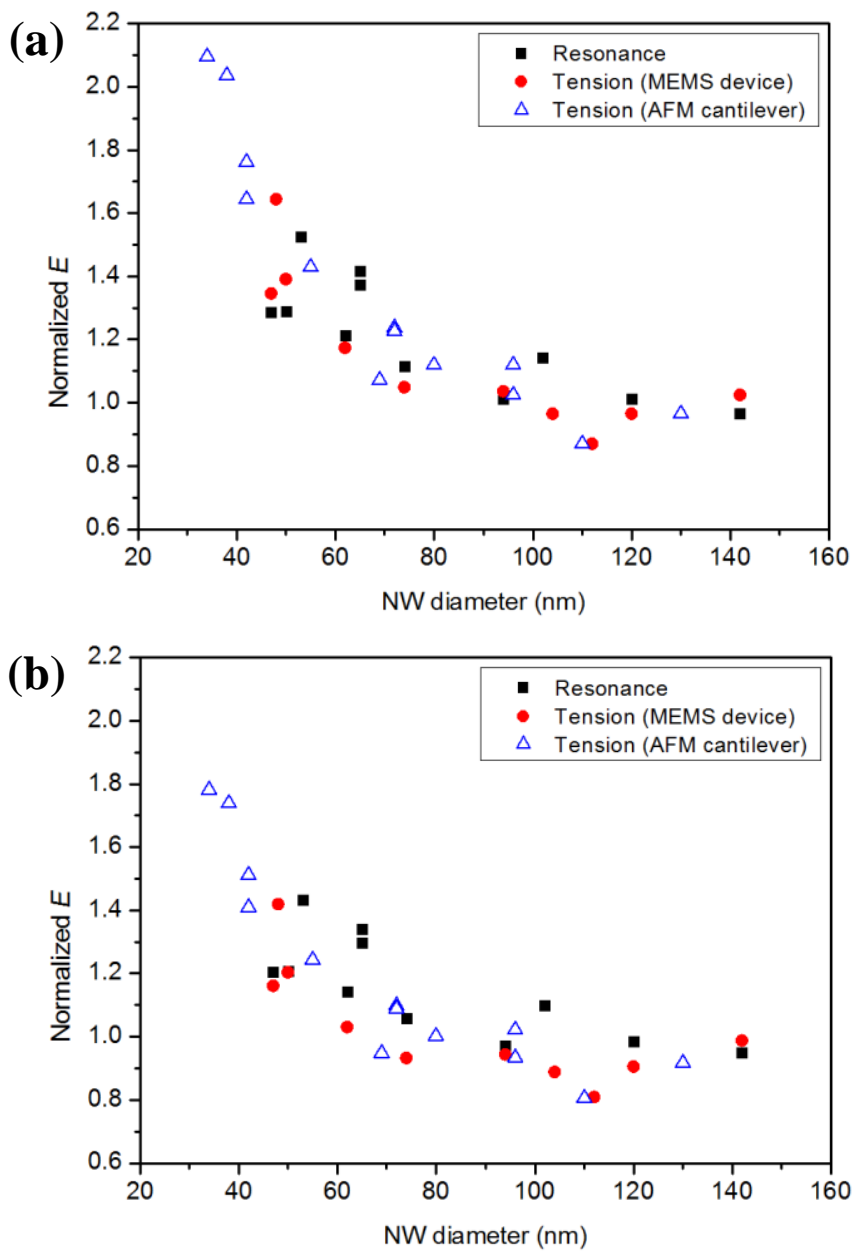

Figure 3. (a) Apparent Young's modulus as a function of NW diameter without any crosssectional shape modification. (b) Modified Young's modulus as a function of NW diameter. The measured Young's modulus was normalized by the bulk modulus of $84 \mathrm{GPa}$. The tension data using AFM cantilever (in blue open triangle) were reported in a previous paper [6]. 
(a)
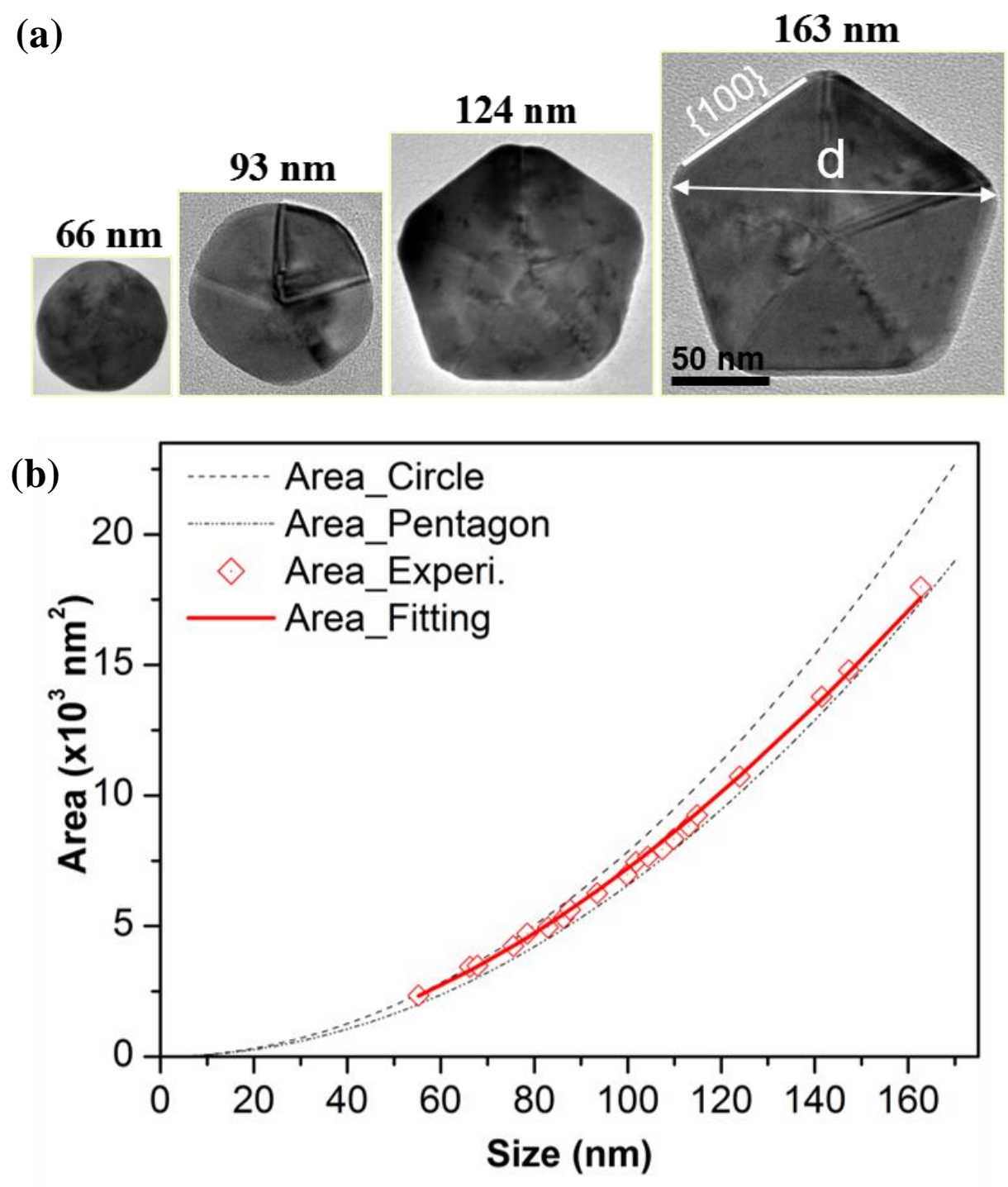

Figure 4. (a) Cross-sectional images of the penta-twinned Ag NWs with different diameters. (b) A plot of cross-sectional area as a function of diameter. 

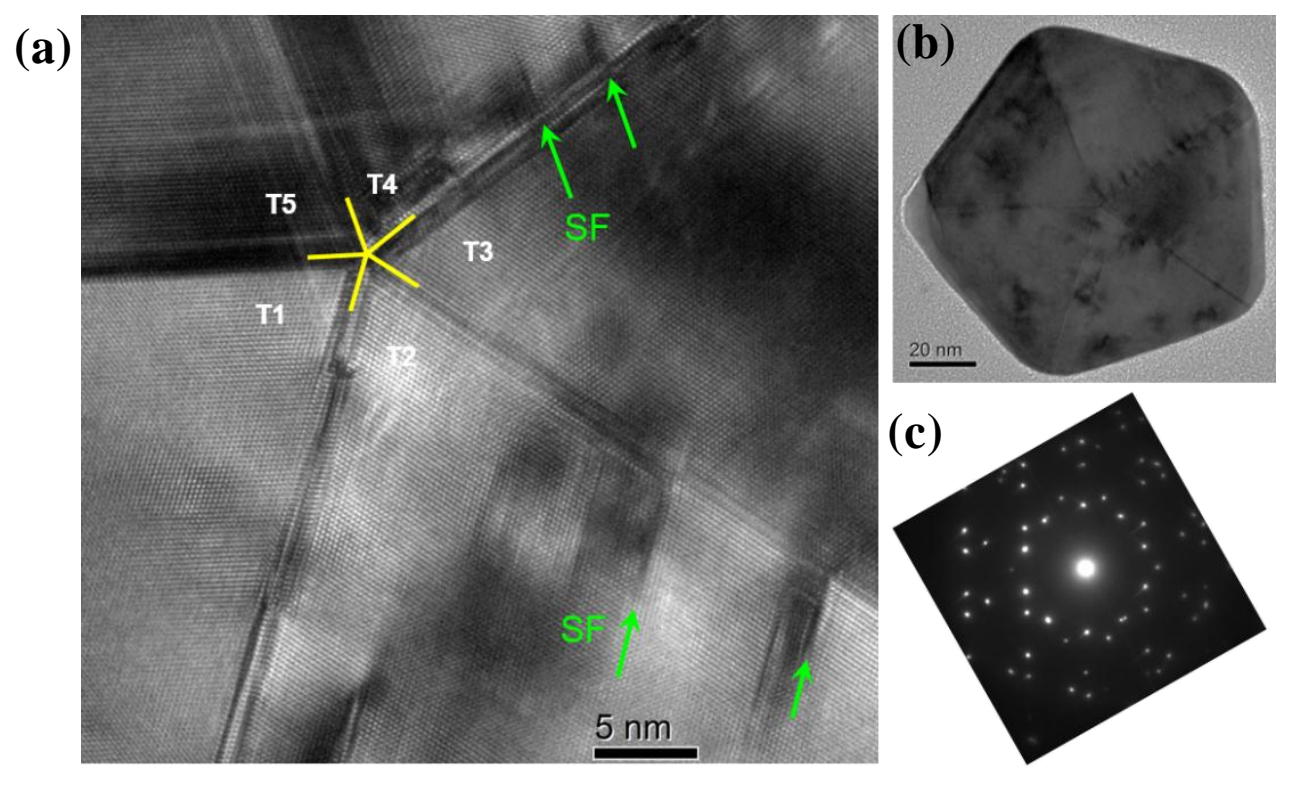

Figure 5. (a) HRTEM image showing defects in the cross-sectional view of a penta-twinned Ag NW. (b) and (c) the corresponding low magnification image and the selected area diffraction pattern in (a), respectively. 